\title{
Avalanche Behavior and Statistical Properties in a Microcrack Coalescence Process
}

\author{
Chunsheng Lu, ${ }^{1,2}$ David Vere-Jones, ${ }^{1}$ and Hideki Takayasu ${ }^{3}$ \\ ${ }^{1}$ Institute of Statistics and Operations Research, School of Mathematical and Computing Sciences, \\ Victoria University of Wellington, Wellington 600, New Zealand \\ ${ }^{2}$ Laboratory for Nonlinear Mechanics of Continuous Media, Institute of Mechanics, Academia Sinica, Beijing 100080, China \\ ${ }^{3}$ Sony Computer Science Laboratory, 3-14-13 Higashi-Gotanda, Shinagawa-ku, Tokyo 151, Japan
}

(Received 19 June 1998)

\begin{abstract}
Fracture owing to the coalescence of numerous microcracks can be described by a simple statistical model, where a coalescence event stochastically occurs as the number density of nucleated microcracks increases. Both numerical simulation and statistical analysis reveal that a microcrack coalescence process may display avalanche behavior and that the final failure is catastrophic. The cumulative distribution of coalescence events in the vicinity of critical fracture follows a power law and the fracture profile has self-affine fractal characteristic. Some macromechanical quantities may be traced back and extracted from the mesoscopic process based on the statistical analysis of coalescence events. [S0031-9007(98)08252-0]
\end{abstract}

PACS numbers: 62.20.Mk, 46.50.+a

The catastrophic fracture in inhomogeneous materials, such as rock and composite, etc. is the result of a sequential process of the nucleation, growth, and coalescence of numerous microcracks or voids created in previously intact media [1]. A classic example is the ductile failure of a smooth bar in tension, where the failure occurs through the nucleation, growth, and coalescence of millions of microscopic voids in the necking of the region. To understand the underlying physical mechanism in such a complex process, one must understand not only the threshold conditions (traditional fracture criteria) that trigger this process, but also the dynamics by which it proceeds.

As an important nonlinear dynamic phenomenon, fracture has been widely attracting much attention from physicists [2]. More recently, several remarkably universal features in fracture process have been discovered. For example, the fracture surface of metals is observed to exhibit fractal characteristics [3], and its topology is usually self-affine with a universal roughness exponent [4]. Studies of acoustic emission from the microfracturing process reveal power-law behavior [5]. All these new discoveries have suggested that a microfracturing process might display criticality or self-organized criticality [6]. Some conceptually simple models related to critical phenomena, such as fractals or multifractals, percolation, and renormalization group, have been applied to fracture studies $[7,8]$. Although significant progress has been achieved, many aspects associated with fracture itself - in particular, a coalescence process which governs the finally catastrophic fracture-are still unclear. In this Letter, we will focus attention on the statistical properties of a coalescence process of microcracks and present a simple statistical model based on numerical simulations.

In order to study the nature of this process, especially the interplay between disorder and dynamical effects at a mesoscopic scale, we have produced a two-dimensional evolution induced catastrophe model by experimental find- ings [9]. In this model, two important processes (microcrack nucleation and coalescence) are involved. The nucleated microcracks are simulated through a random distribution function which is often closely related to the distribution of microstructures, such as second-phase particles, inclusions, and flaws, etc. The coalescence condition between two neighboring microcracks can be approximately determined by mechanical calculation. Here, as an example, we consider a simple dynamics of damage evolution, i.e., a load-sharing principle where the load supported by a broken part is shared by its two neighboring intact parts. The average stress on an intact segment is $\bar{\sigma}=(1+\bar{c} / d) \sigma_{0}$, where $\sigma_{0}$ is the nominal stress, $\bar{c}$ is the average length of two microcracks, and $d$ is the Euclidean distance between the closest tips of two neighboring microcracks. We suppose that two neighboring microcracks will coalesce when the average stress $\bar{\sigma}$ attains a critical value $\sigma_{c}$, then the critical coalescence condition can be written as

$$
r=\frac{d}{\bar{c}}=\frac{\sigma_{0}}{\bar{\sigma}-\sigma_{0}} \leq r_{c}=\frac{\sigma_{0}}{\sigma_{c}-\sigma_{0}} .
$$

Here, the probability for microcrack coalescence is independent of the angle between the orientation of the cracks and the line joining their tips. The length of each coalesced crack is approximately represented by its projection normal to the loading direction. The time evolution of stress can be indirectly calculated from the crack density.

More details on the model were discussed in Ref. [9]. The dynamics used in numerical simulations can be briefly summarized as follows:

(1) A newly nucleated microcrack is added randomly in the lattice, with the horizontal orientation and the length from a given size distribution.

(2) Check the coalescence condition for the newly nucleated crack with all preexisting cracks, in turn, starting from the closest pair. If the left tip of the new crack lies 
below or above the right tip of an existing crack (or vice versa), it is supposed that the new crack is shielded by the old one, and coalescence would not occur.

(3) Repeat procedure (2) until there are no more cracks satisfying the condition and, at the same time, there is not a crack spanning the whole lattice. Then, return to procedure (1).

A typical evolution process in a $1000 \times 1000$ lattice is shown in Fig. 1, where the critical parameter $r_{c}=1$ and a uniform length distribution of nucleated microcracks with the mean length equal to 2 (lattice units) are used in the simulation. It is obvious to see there are three different phases (stages) in this process. (i) Initial phase: New (small) events appear too sparse to cause coalescence, and the number of microcracks increases linearly with time; (ii) stable phase: a significant proportion of new events fall close enough to an existing crack tip to cause coalescence. Such a coalescence has two effects: (a) It increases the zone of influence around crack tip and so increases the chance of a coalescence event, perhaps a large one, and (b) it increases the area shielded from the coalescence. In this case, if a new event appears in the shielded region, it does not coalesce. These two effects balance producing a near-stable regime; and (iii) critical phase: After some time, the zones of influence extend and overlap to such an extent that a newly nucleated microcrack (small fluctuation) will trigger a cascade coalescence of microcracks and result in a catastrophic failure (avalanche). We could not predict, a priori, the position where the final fracture appears (see Fig. 1).

The variation of coalescence events versus time can be distinguished from the evolution process, as shown in Fig. 2. Clearly, coalescence events occur stochastically in both space and time as the number density of nucleated microcracks increases in the system. As we usually see and expect $[10,11]$, the cumulative distribution of coalescence events in the vicinity of critical failure follows a power-law distribution (see Fig. 3). At the same time, we also calculated the roughness of fracture interfaces. For a given configuration, the roughness of a fracture profile is defined as $w=\left\langle\max h_{i}(x)-\min h_{i}(x)\right\rangle$, where $h_{i}(x)$ is the inter-

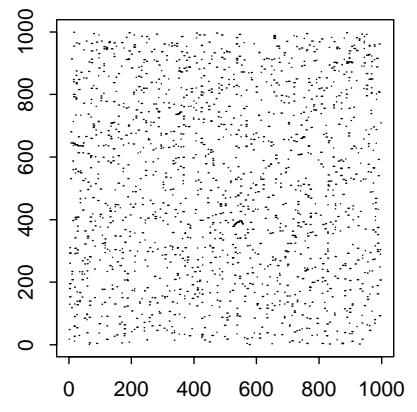

(a)

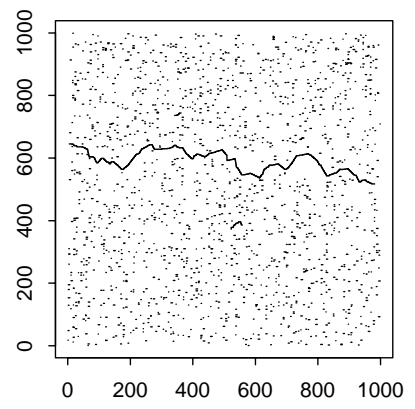

(b)
FIG. 1. Patterns of a microcrack coalescence process in a $1000 \times 1000$ lattice. (a) Just before fracture and (b) a newly nucleated microcrack triggers the catastrophic fracture. face position of a fracture profile. As presented in Fig. 4, it is clearly seen that there is a scaling law, $w \sim L^{\zeta}$ with the exponent $\zeta=0.67 \pm 0.02$, which is well consistent with experimental results [4]. In other words, the fracture surface has a self-affine fractal characteristic.

Although an exact analysis of this process is very difficult and even impossible, some general features can be extracted [12]. As discussed above, a newly nucleated microcrack will increase the local stress, especially the stress at its tip. Otherwise, the coalescence of two neighboring microcracks will transfer and redistribute the stress in the system. Here, the stress level (the number density of nucleated microcracks) is a key parameter which controls the probability of a coalescence event occurrence. For simplicity and as a first attempt, we approximately consider that the stress level $\sigma(t)$ increases deterministically between two coalescence events and releases stochastically as a scalar Markov process when a coalescence event occurs. The evolution of stress level versus time follows the equation

$$
\sigma(t)=\sigma(0)+\rho t-\sum_{i: t_{i}<t} S_{i}(t),
$$

where $\sigma(0)$ is the initial stress level, $\rho$ is the constant loading rate from external force or displacement, and $S_{i}$ is the stress release associated with the $i$ th coalescence event at time $t_{i}$. It is called a stress release model (SRM) [13]. Here, the stress release value $S_{i}$ is supposed to be proportional to the length of a coalesced crack. On the other hand, we can also consider the coalescence process from an energy viewpoint by replacing $S_{i}$ in Eq. (2) with $E_{i} \sim S_{i}^{2}$ (i.e., the so-called energy release model).

The probability intensity of a coalescence event is controlled by the risk function $\Psi(\sigma)$, which is defined by the conditional probability of a coalescence event occurring in the time interval $(t, t+\delta t)$ given that $\sigma(t)$ has the current value $\sigma$. Generally speaking, it is very difficult to model fully, but to a first approximation, the SRM

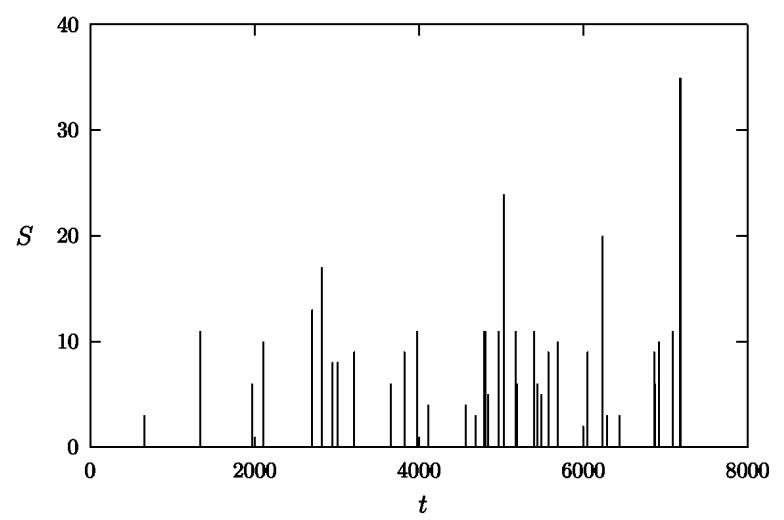

FIG. 2. The magnitude of coalescence events as a function of time in the evolution process shown in Fig. 1. Here, the magnitude of a coalescence event is proportional to its length. 
$P(>S)$

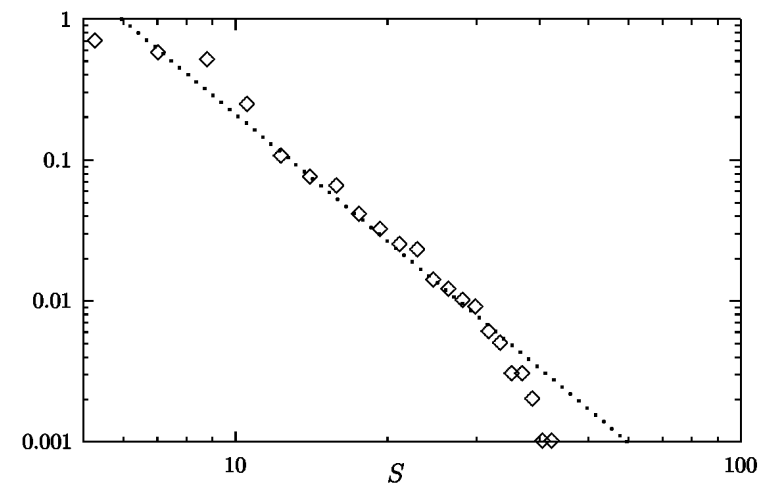

FIG. 3. The cumulative distribution of coalescence events (over around 30 random simulations) with magnitude greater than $S$, where the slope of the dotted line is -3.0 .

can be used to model at least the first and second phases as mentioned above. The simplest form of $\Psi(\sigma)$ is taken as an exponential function $\Psi(\sigma)=\exp (\mu+\nu \sigma)$, where $\mu$ and $\nu$ are constants with $\nu$ indicating the sensitivity to risk. This is a convenient compromise between time-predictable and purely random (Poisson) processes [14].

The key for statistical analysis is that the coalescence events in the evolution process can be treated as a point process in time-stress space with the conditional intensity function

$$
\lambda(t)=\Psi[\sigma(t)]=\exp \left(\alpha+\nu\left[\rho t-\sum_{i: t_{i}<t} S_{i}(t)\right]\right),
$$

where $\alpha[=\mu+\nu \sigma(0)], \nu$, and $\rho$ are the parameters to be fitted, and the distribution of the stress release $S_{i}$ in a coalescence event is independent of the stress level itself. Estimates of these parameters can be found by maximizing the log-likelihood function

$$
\log L=\sum_{i=1}^{N} \log \lambda\left(t_{i}\right)-\int_{0}^{T} \lambda(\mu) d \mu
$$

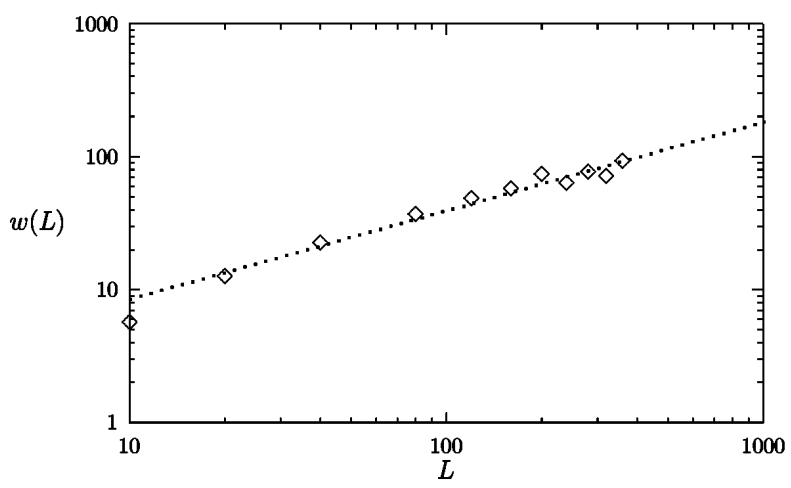

FIG. 4. Log-log plot of the fracture profile roughness versus the system size $L$ (see Fig. 1), where the slope of the dotted line is $\frac{2}{3}$. where $T$ is the length of observation interval and $N$ is the total number of coalescence events observed in that interval [15].

The choice of models is based on the Akaike information criterion (AIC), which is defined as AIC $=-2 \log \hat{L}+$ $2 k$, where $\log \hat{L}$ is the maximum likelihood for a given model and $k$ is the number of parameters to be fitted in this model [16]. This represents a rough way of compensating for the effect of adding parameters, and is a useful heuristic measure of the relative effectiveness of different models. For example, the stress release model with three parameters as against the Poisson model with only one or the Poisson with exponential trend with two, should demonstrate a significantly better fit to justify the additional parameters. The best model is selected for which AIC has the smallest value [17]. However, we should notice that the AIC value obtained here is used as a rough guide only, since the amount of coalescence events is not very large and the distribution of the log-likelihood is nonstandard.

Analytical results for the microcrack coalescence process, as shown in Figs. 1 and 2, indicate that the stress release model fits the data better than both the Poisson model $[\Delta \mathrm{AIC}=\mathrm{AIC}(\mathrm{SRM})-\mathrm{AIC}($ Poisson $)=-4.99]$ and the Poisson model with trend $[\Delta \mathrm{AIC}=\mathrm{AIC}(\mathrm{SRM})-$ AIC(Poisson with trend) $=-1.58]$. This means that the coalescence process is not fully random. Using the fitted parameters, we can reproduce the change of the total stress versus time in the system, as shown in Fig. 5. A near-critical stable state is observed in the vicinity of critical fracture where the interaction between enlarging and shielding effects in the coalescence process is balanced. As recent research shows, if some similar mechanism, such as plasticity, is introduced, critical steady state can be also observed in microfracturing phenomena [18]. Using the same approach, we can check the problem of whether or not a final fracture surface is governed by minimum energy [19]. Unfortunately, the difference of AIC values between stress release model and energy release model is

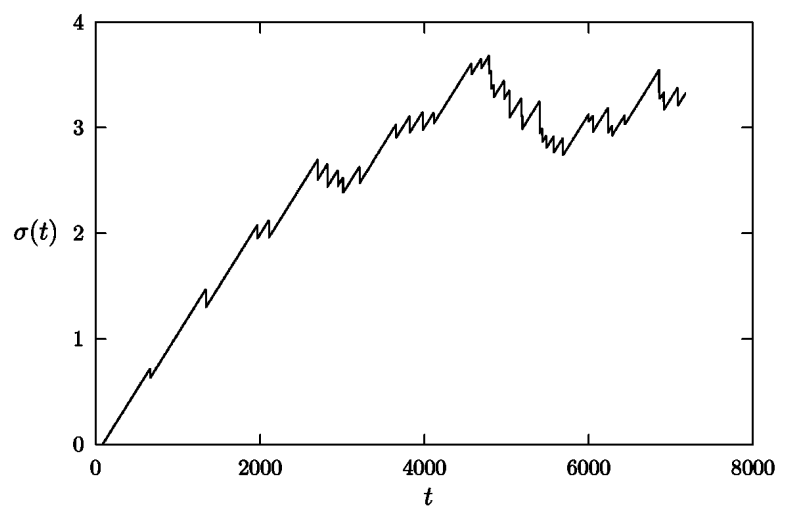

FIG. 5. The total stress in the system versus time is reproduced by the fitted parameters in the stress release model, where $\alpha=-6.98, \rho=0.075$, and $\nu=0.015$. 
too small to be distinguished by the present simulation model.

All the results mentioned above indicate that there are some hints of long-range interaction in a microcrack coalescence process. This can be highlighted through a natural extension of the present SRM model. We can divide the system into several sections and consider the interplay between different sections as follows:

$$
\lambda_{i}(t)=\exp \left\{\alpha_{i}+\nu_{i}\left[\rho_{i} t-\sum_{j} \theta_{i j} S^{(j)}(t)\right]\right\},
$$

where $S^{(j)}(t)$ is the accumulative stress release in section $j$ over the period $(0, t)$, and the coefficient $\theta_{i j}$ represents the fixed proportion of stress drop, initiated in the $j$ th section, which is transferred to the $i$ th section and $\theta_{i i}=$ 1. If $\theta_{i j}=0$ for all $i \neq j$, it is reduced to the simple form [see Eq. (3)]. Through different combinations of the parameters in Eq. (5) and the difference in AIC values obtained, we can deeply understand the underlying physics in this process.

The stress release model proposed here is not understood as a best model, but as a first attempt to find a model that allows the internal state of the specimen to be judged from external orientation. In this model, the risk function as well as the stress calculation is oversimplification. The present description may be suitable to the mesoscopic level where the microcrack number density is a key parameter. However, this assumption has to be modified if an accurate simulation of the properties of real materials is required. The attempts to produce a more adequate model are on progress and will be reported in another paper.

In summary, a new statistical model for the statistical analysis of a microcrack coalescence process is presented in this Letter. Both numerical simulations and statistical analysis show that there is an avalanche behavior in this process. It can be explained as a critical phenomenon and the competition between enlarging and screening (or relaxation) plays an important role in the evolution process. Based on the statistical analysis of the data from simulations, some macromechanical behaviors can be traced back and finally extracted from the mesoscopic evolution process. As one of direct implications, the present method can be easily applied to the statistical analysis of acoustic emission signals from the microfracturing process.

This work is supported in part by the Marsden Fund of the Royal Society of New Zealand. Several routines in the Statistical Seismology Library (SSLib) devised by D. Harte et al. were used in the statistical analysis. One of the authors (C. Lu) would like to thank Y. Bai for many valuable discussions and support from the Japan Society for the Promotion of Science while he was a postdoctoral fellow at Tohoku University.
[1] D. R. Curran, L. Seaman, and D. A. Shockey, Phys. Rep. 147, 253 (1987), and references therein.

[2] J. Fineberg, S. P. Gross, M. Marder, and H. L. Swinney, Phys. Rev. B 45, 5146 (1992); M. Marder, Nature (London) 381, 275 (1996).

[3] B. B. Mandelbrot, D. E. Passoja, and A. J. Paullay, Nature (London) 308, 721 (1984); R. Cahn, Nature (London) 338, 201 (1989).

[4] E. Bouchard, G. Lapasset, and J. Planès, Europhys. Lett. 13, 73 (1990); K. J. Måløy, A. Hansen, E. L. Hinrichsen, and S. Roux, Phys. Rev. Lett. 68, 213 (1992).

[5] A. Petri, G. Paparo, A. Vespignani, A. Alippi, and M. Costantini, Phys. Rev. Lett. 73, 3423 (1994).

[6] P. Bak, C. Tang, and K. Wiesenfeld, Phys. Rev. Lett. 59, 381 (1987); Phys. Rev. A 38, 364 (1988); P. Bak and C. Tang, J. Geophys. Res. 94, 15635 (1989).

[7] H. Takayasu, Fractals in the Physical Sciences (Manchester University Press, Manchester, 1990).

[8] Statistical Models for the Fracture of Disordered Media, edited by H. J. Herrmann and S. Roux (North-Holland, Amsterdam, 1990).

[9] Y. Bai, C. Lu, F. Ke, and M. Xia, Phys. Lett. A 185, 196 (1994); C. Lu, F. Ke, Y. Bai, and M. Xia, Sci. China A 38, 462 (1995).

[10] G. Caldarelli, F. D. Di Tolla, and A. Petri, Phys. Rev. Lett. 77, 2503 (1996).

[11] A. Garcimartin, A. Guarino, L. Bellon, and S. Ciliberto, Phys. Rev. Lett. 79, 3202 (1997).

[12] M. Xia, F. Ke, J. Bai, and Y. Bai, Phys. Lett. A 236, 60 (1997).

[13] D. Vere-Jones, J. Phys. Earth 26, 129 (1978); X. Zheng and D. Vere-Jones, Pure Appl. Geophys. 135, 559 (1991).

[14] If the solid media had an exact critical strength, $\Psi(\sigma)$ would have to be zero until $\sigma$ reached the critical strength, and infinite beyond it. By contrast, a finite constant value of $\Psi(\sigma)$ corresponds to a pure random process in which the occurrence of events is independent of the stress level. Thus, an exponential function is a convenient compromise between these two extreme cases.

[15] Here, the independence assumption allows the likelihood to be written as the product of two terms, a likelihood for the times, taking the observed stress drops as given, and a likelihood for stress drops, which reduces to the standard form for a set of independent, identically distributed values. For detail discussion, see D. Daley and D. VereJones, An Introduction to the Theory of Point Processes (Springer, Berlin, 1988).

[16] Y. Sakamoto, M. Ishiguro, and G. Kitagawa, Akaike Information Criterion Statistics (Reidel, Dordrecht, 1983).

[17] In typical cases, model differences which would be significant at around the 5\% level correspond to differences in AIC values of around 1.5 or 2 .

[18] S. Zapperi, P. Ray, H.E. Stanley, and A. Vespignani, Phys. Rev. Lett. 78, 1408 (1997); S. Zapperi, A. Vespignani, and H. E. Stanley, Nature (London) 388, 658 (1997).

[19] G. George Batrouni and A. Hansen, Phys. Rev. Lett. 80, 325 (1998); V. I. Räisänen, E. T. Seppala, M. J. Alava, and P. M. Duxbury, Phys. Rev. Lett. 80, 329 (1998). 